Alina Ibbeken*, Pragathi Gurumurthy, Fenja Zell, Christina Hagen, Martin A. Koch, and Thorsten M. Buzug

\title{
MRI flow measurements with a flexible stenosis phantom
}

https://doi.org/10.1515/cdbme-2019-0142

\begin{abstract}
Obstructive sleep apnea (OSA) is a common sleep disorder caused by the collapse of the upper airway during sleep due to a shift of soft tissues. To optimise the treatment, one needs a better understanding of the patient-specific causes of OSA. As a first step, a flexible tube with a stenosis was manufactured, representing a simple model of the pharynx geometry. The phantom has been used to carry out magnetic resonance imaging (MRI) flow sensitive measurements, to investigate the flow conditions that lead to the collapse. This contribution is concerned with the comparison and analysis of the flow and phantom deformation using varying boundary conditions, such as the Reynolds number and transmural pressure.
\end{abstract}

Keywords: Obstructive sleep apnea, computational fluid dynamics simulation, experimental validation, flow measurement, deformation measurement, phase contrast magnetic resonance imaging

\section{Introduction}

Obstructive sleep apnea (OSA) is a common sleeping disorder caused by the repetitive complete or partial obstruction of the upper airway due to a shift of the soft tissue. Besides a significantly reduced quality of sleep, OSA might lead to a whole range of secondary pathologies like diabetes, hypertension and cardiovascular disease [1]. For a better understanding of the airflow characteristics and tissue conditions that lead to the obstruction, numerical simulations can be used to calculate flow patterns and deformation behaviour. To ensure reliability of the simulations, independent validation is essential. Especially in the field of angiography, 2D phase-contrast (PC) magnetic resonance imaging (MRI) is often used for quantifying flow phenomena. Nevertheless, applying 2D PC-MRI to OSA is a relatively new approach. While quite a few studies used particle image velocimetry [2,3], pressure transducers and hot wire anemometry [4] to study flow behaviour in the upper air-

${ }^{*}$ Corresponding author: Alina Ibbeken, Institute of Medical Engineering, Universität zu Lübeck, Lübeck, Germany, e-mail: ibbeken@imt.uni-luebeck.de

Pragathi Gurumurthy, Fenja Zell, Christina Hagen, Martin

A. Koch, Thorsten M. Buzug, Institute of Medical Engineering, Universität zu Lübeck, Lübeck, Germany way, to the best of our knowledge so far no studies exist that use 2D PC-MRI. There are also very few studies in OSA research that use flexible phantoms to examine wall deformation occuring during the flow time course [5]. Using 2D PC-MRI as an investigating tool for flow fields in flexible phantoms offers the main advantage that both data of the 3D flow field and of the resulting deformation are available. In this study, first 2D PC-MRI measurements with a simple flexible phantom will be conducted to measure flow field and deformation simultaneously. A flexible stenosis phantom was produced using additive manufacturing from silicone (Sylgard 184 Silicone Elastomere, Dow Corning). Four different 2D PC-MRI measurements were carried out with varying inlet conditions, e.g. transmural pressure and flow velocity. A glycerol/water mixture was used as MRI-visible flow medium. The flow behaviour can be assumed to be similar to the flow behaviour of air if the same Reynolds number is used. The Reynolds number is defined as $R e=\frac{v \cdot D}{\nu}$ where $v$ is the flow velocity, $D$ is the diameter and $\nu$ is the kinematic viscosity [6]. As result of the measurements, the 3D flow field and the cross sectional area of the phantom were obtained. A measure of the deformation was calculated by normalising the area measurements with a zero measurement, in which the phantom geometries were measured without any fluid, meaning no flow and zero transmural pressure.

\section{Material and Methods}

\subsection{Phantom fabrication}

A flexible flow phantom was cast from silicone (Sylgard 184). The phantom production aimed to mimic the model used by Geoghegan [2]. For the casting process, a water-soluble polyvinyl alcohol (Ultimaker PVA, Ultimaker, Geldermalsen, Netherlands) casting mold was manifactured with a 3D printer (Ultimaker 2, Ultimaker, Geldermalsen, Netherlands). The inner diameter of the phantom was $20 \mathrm{~mm}$ with a wall thickness of $1.3 \mathrm{~mm}$. A $50 \%$ rotational symmetric diameter stenosis, $40 \mathrm{~mm}$ in length, is part of the phantom. The Young's modulus of Sylgard 184, which was measured with a tensile test (AllroundLine Z020 TN, Zwick/Roell, Ulm, Germany), is $1.2 \times 10^{6} \mathrm{~N} \mathrm{~m}^{-2}$. Further information about the phantom 
fabrication can be found in [7]. An image of the phantom is depicted in Figure 1.

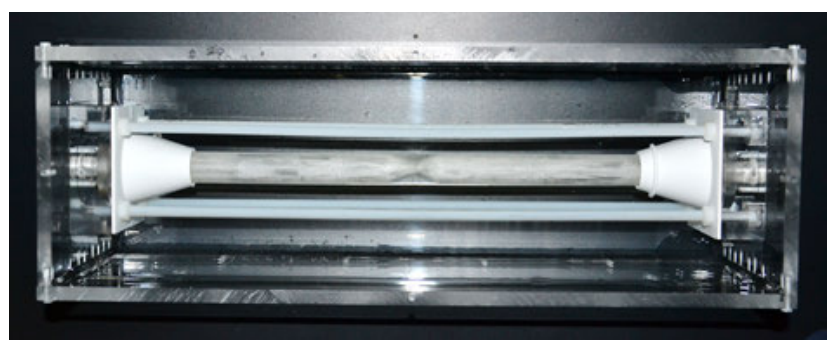

Fig. 1: Stenosis phantom.

\subsection{PC-MRI measurements}

To study the flow and deformation behaviour of the phantom with varying inlet conditions (e.g. inlet velocity, transmural pressure) 2D PC-MRI measurements were conducted. Common values used in other flow studies regarding upper airway flow range approximately in between $\mathrm{Re}=500$ and $\mathrm{Re}$ $=1000$ for light breathing [8, 9]. An MRI compatible flow pump (CardioFlow-5000MR, Shelley Medical Imaging Technologies, London, Ontario Canada) was used to provide the Reynolds numbers for steady flow of 550, 900 and 1000, corresponding to mean inlet flow velocities of $16 \mathrm{~cm} \mathrm{~s}^{-1}$, $26 \mathrm{~cm} \mathrm{~s}^{-1}$ and $29 \mathrm{cms}^{-1}$. As recommended for the pump a glycerol/water mixture was used as flow medium with $55 \%$ glycerol mass fraction. The transmural pressure, which is defined as the pressure difference between inner and outer pressure, was set to $8 \mathrm{kPa}$ and $11 \mathrm{kPa}$. It was modified by changing the inner pressure due to varying hydrostatic pressure. The outer pressure was generated by placing the phantom in a water basin and was not changed during the experiments.

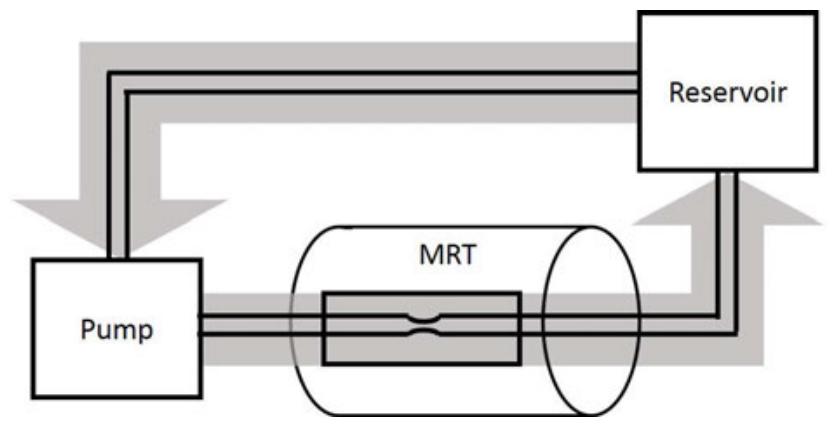

Fig. 2: Experimental flow diagram.

The experiments were performed on a $3 \mathrm{~T}$ MR system (Ingenia, Philips, Amsterdam, Netherlands) with a body coil using a RF gradient echo sequence. Further MR parameters include the repetition time TR of $10 \mathrm{~ms}$, the echo time TE of $6 \mathrm{~ms}$, a flip angle of $7^{\circ}$, a nominal in-plane resolution of 0.89 $\mathrm{x} 0.89 \mathrm{~mm}^{2}$ and a slice thickness of $1 \mathrm{~mm}$. A flow diagram of the experimental setup is shown in Figure 2.

\subsection{Area measurement}

The area of the phantom's cross section in the MR images was determined using Matlab. A threshold and a seed point were selected manually as input. A region-growing algorithm with thresholding was then used for segmenting the interior area. The area was calculated for each slice perpendicular to the flow (Figure 3).
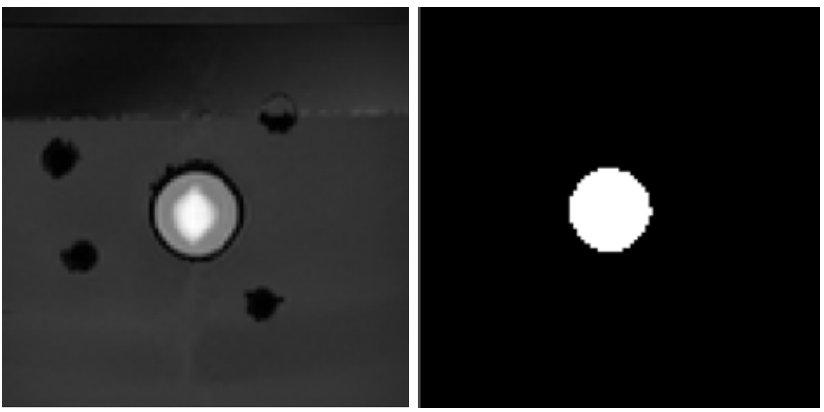

Fig. 3: Cross sectional area measurement. By selecting a threshold in the magnitude image (left) the area of the phantom can be determined automatically (right). The wall of the phantom is depicted in the left image as a black circle.

\section{Results}

\subsection{Velocity measurement}

Figure 4 shows a comparison of the resulting velocity flow fields for varying inlet conditions. In all four measurements the velocity increases significantly as the flow passes the stenosis. Behind the stenosis, flow separation can be observed and a jet is formed. The jet length decreases with an increase of the inlet velocity. For higher inlet velocities $(\operatorname{Re}=900, \operatorname{Re}=1000)$, negative flow velocities occur lateral to the jet. An increase of the transmural pressure leads to slightly higher velocities inside the jet as well as to a slightly reduced jet length. 


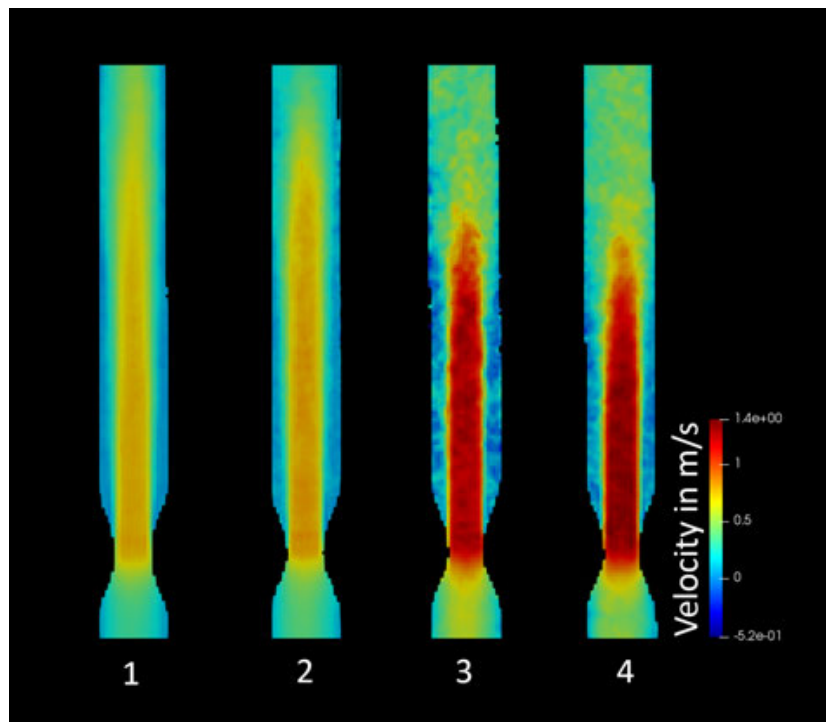

Fig. 4: Velocity fields recorded with 2D PC-MRI in $\mathrm{m} \mathrm{s}^{-1}$. Inlet conditions from left to right: $1: \operatorname{Re}=550, \Delta p=8 \mathrm{kPa} ; 2: \operatorname{Re}=$ $550, \Delta \mathrm{p}=11 \mathrm{kPa}$; $: \operatorname{Re}=900, \Delta \mathrm{p}=8 \mathrm{kPa} ; 4: \operatorname{Re}=1000, \Delta \mathrm{p}=$ $8 \mathrm{kPa}$.

\subsection{Area measurement}

The upper diagram in Figure 5 shows the absolute values of the cross sectional area measurement perpendicular to the flow in $\mathrm{mm}^{2}$. The zero position corresponds to the position of the minimum diameter of the stenosis. A zero measurement is used for normalisation. Therefore, a CT image of the phantom without any fluid inside and with no transmural pressure was acquired. One can see that the phantom expands if filled with fluid. In the lower diagram of Figure 5, the normalised cross sectional area measurement is shown. One can see that for all measurements upstream of the minimum diameter position the phantom expands whereas downstream of the minimum diameter position the phantom contracts.

\section{Discussion}

The velocity measurements show that increasing inlet velocity or transmural pressure lead to a higher jet velocity as well as to a shorter jet length. This is due to stronger lateral turbulences that develop with higher velocity and lead to an energy loss of the jet. Thus, the jet dissipates faster.

Even though just a small change in the absolute cross sectional area due to increasing inlet velocity or transmural pressure is detected, the normalised cross sectional area measurement shows a significant change of area close to the stenosis. At the inlet upstream of the minimal diameter, the phantom is expanded. This is probably due to the increasing constriction that might lead to increased wall shear stress and thus to a deformation. Downstream of the minimal diameter at the outlet, a narrowing of the phantom can be observed. This narrowing might originate in a negative pressure that develops due to the emerging turbulences behind the stenosis. The amount of change is highest for the measurement with the fastest velocity $(\operatorname{Re}=1000)$ which meets the expectations. However, the values of the measurement with $\mathrm{Re}=900$ show less deformation than the values of the measurement with $\operatorname{Re}=550$. However, the difference between these two results is on the same order of magnitude as the fluctuations in Figure 5 (bottom). The fluctuations are likely to be caused mainly by image noise and due to a certain measurement uncertainty caused by the image resolution limit of $0.89 \times 0.89 \mathrm{~mm}^{2}$. Also the segmentation uncertainty influences the results. As the thresholding method adds only entire image pixels with $0.79 \mathrm{~mm}^{2}$ to the segmentation, an exact area detection is not possible, as pixels in the edge region might be segmented incorrectly. Concluding one could say that the deformation of this phantom with the chosen velocities is too small to accurately detect it with the used image resolution. Hence, further experiments are needed to investigate the interdependence between inlet velocity and deformation. A possible improvement in the methodology would be to normalise the data with measurements that were taken with the same transmural pressure instead of normalising data with a CT measurement taken without fluid and thus zero transmural pressure.

\section{Conclusion}

In this study, 2D PC-MRI measurements with a flexible phantom were carried out. As expected, the flow velocities increase significantly upon passing the stenosis and a jet develops. The corresponding deformation measurements show a significant change of area in the region of the stenosis. Thus this study marks, to our knowledge, the first study that demonstrates the feasibility and great potential of using 2D PC-MRI to measure flow and deformation behaviour of flexible phantoms simultaneously. Consequently, 2D PC-MRI can be used for the validation of computational fluid dynamics simulations with fluid-structure interaction.

Further improvements of the methodology is required, since some of the results are not in line with our expectation, e.g. the deformation comparison between the measurement with $\mathrm{Re}=$ 550 and $\operatorname{Re}=900$. However, in general, it has been shown that 2D PC-MRI is well suited for the purpose of future studies in which flow behaviour and deformation behaviour of flexible upper airway phantoms shall be examined. 

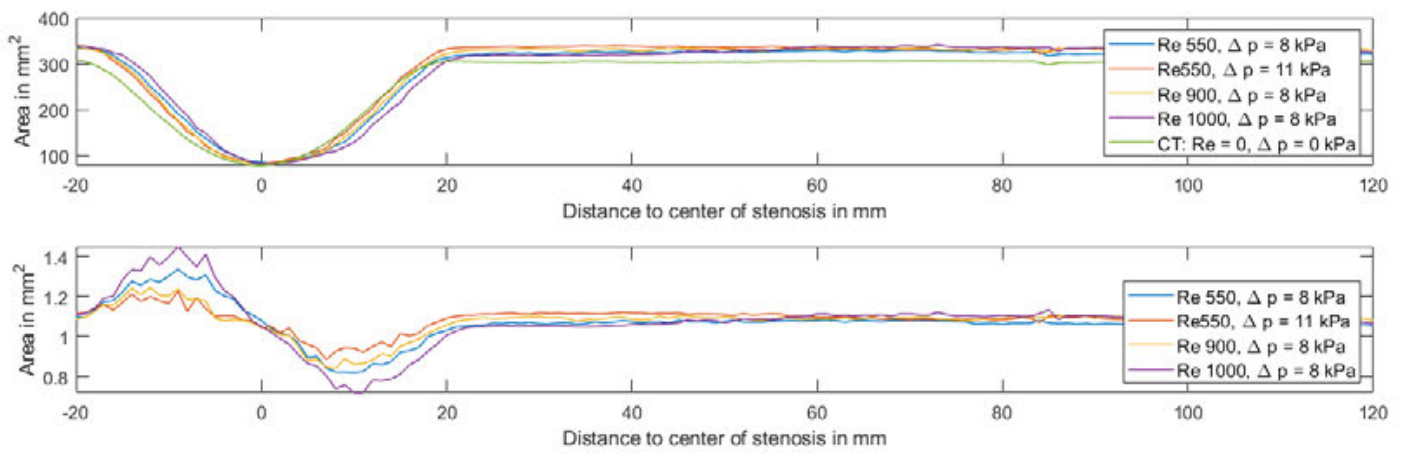

Fig. 5: Absolute (upper diagram) and normalised (lower diagram) cross sectional area measurements in $\mathrm{mm}^{2}$. For the normalisation the CT zero measurement (no fluid, no transmural pressure) was used. The horizontal axis defines the distance to the minimal diameter of the stenosis in $\mathrm{mm}$.

\section{Acknowledgement}

tational fluid dynamics; Medical Engineering \& Physics; 29 637-651; 2007.

This work has been sponsored by the German Federal Ministry of Education and Research (BMBF Grant Number 13GW0276B).

\section{References}

[1] Eckert DJ, Malhotra A. Pathophysiology of adult obstructive sleep apnea. Proceedings of the American Thoracic Society; 493-504; 2008.

[2] Geoghegan PH. In Vitro Experimental Investigation Into the Effect of Compliance on Models of Arterial Hemodynamics. Dissertation. University of Canterbury; New Zealand; 2012.

[3] Spence CJT. Experimental investigations of airflow in the human upper airways during natural and assisted breathing. Dissertation. University of Canterbury; New Zealand; 2011.

[4] Mylavarapu G, Murugappan S, Mihaescu M, Kalra M, Khosla S, Gutmark E. Validation of computational fluid dynamics methodology used for human upper airway flow simulations. Journal of Biomechanics; 42:1553-9; 2009.

[5] Cheng M, Phaneuf C, Tovar B. Sleep apnea simulation: An experimental assessment of pharyngeal collapse. Department of Medical Engineering; The Cooper Union for the Advancement of Science and Art; 2008.

[6] Bschorer S. Technische Strömungslehre - Lehr- und Übungsbuch; Springer Fachmedien Wiesbaden; 2017.

[7] Ibbeken A. Konstruktion einer Strömungsanlage und Strömungsmessungen an einem flexiblen Stenose-Modell. Master thesis. Univsität zu Lübeck; Germany; 2018.

[8] Wen J, Inthavong K, Tian ZF, Tu JY, Xue CL, Li CG. Airflow Patterns in Both Sides of a Realistic Human Nasal Cavity for Laminar and Turbulent Conditions; 16th Australasian Fluid Mechanics Conference; 2007.

[9] Jeong SJ, Kim WS, Sung SJ. Numerical investigation on the flow characteristics and aerodynamic force of the upper airway of patient with obstructive sleep apnea using compu- 